Three compiled photos illustrate the tension between phylogeny (the branching pattern in the background) and ontogeny (the fish embryo at the phylotypic stage) The central field that links phylogeny and ontogeny represents the phylostratigraphic approach. Image courtesy of V. Solin (http://www. proces15.com) and A. Hartl (http://nautilusfilm.com).

\title{
DEVELOPMENT
}

\section{Hourglass theory gets molecular approval}

It was first observed two centuries ago that many animal embryos converge on a similar form during development, only to diverge afterwards. This temporal 'waist' in the so-called hourglass model of embryonic development is known as the 'phylotypic stage', and it is thought - controversially, given that it is based on subjective morphological comparisons - to represent an evolutionary conserved period. Now, two transcriptomics studies give this

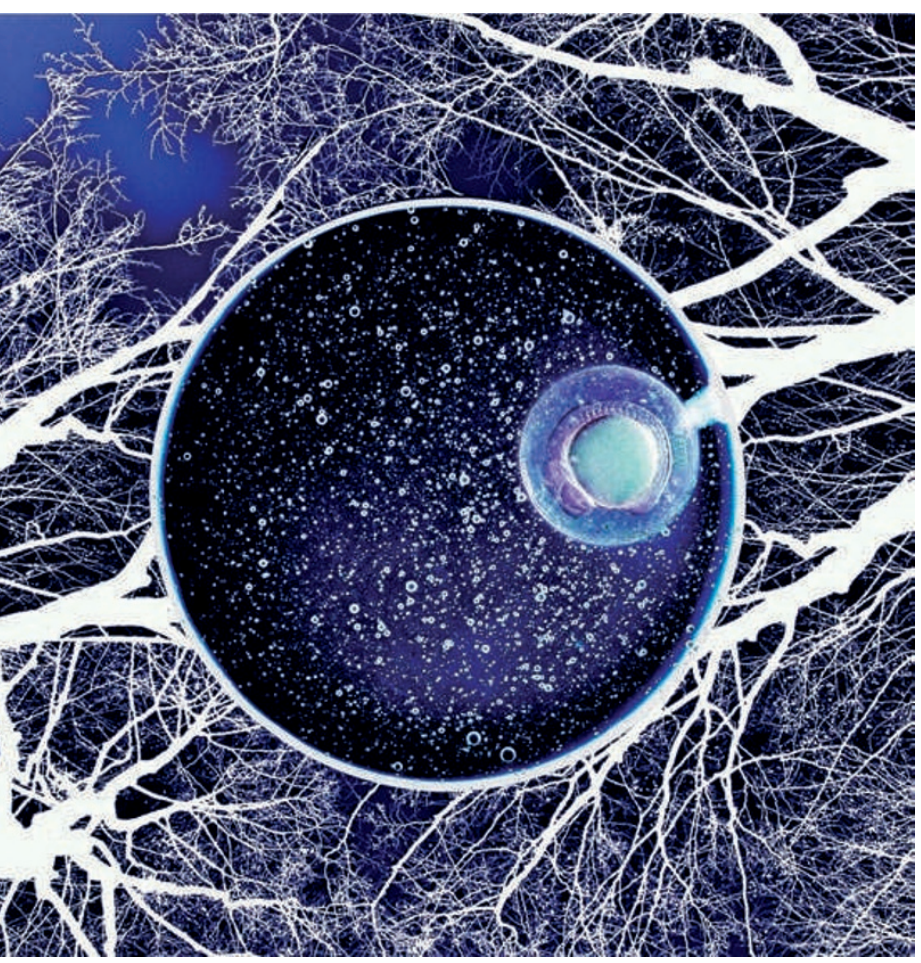

theory strong molecular support by showing that genes active during this period are more ancient and more highly conserved in their expression.

Kalinka, Varga and colleagues directly tested the hourglass model by comparing genome-wide expression profiles across embryonic development in six species of Drosophila. By generating a DNA microarray time-course for over 3,000 orthologous genes, they show that variation in temporal gene expression across species is minimal at the phylotypic stage and is greater before and after that point. Modelling suggests that this pattern is best explained by selective constraint, which seems to be strongest at the phylotypic stage. The genes whose expression follows the hourglass pattern are also most likely to be involved in core developmental processes.

In a related study, Domazet-Lošo and Tautz combined transcriptomics and evolutionary developmental genomics to connect morphological novelties to the evolutionary age of genes that are expressed at different developmental stages. They used a previously described approach known as 'phylostratigraphy' to divide the genome into gene classes, each of which encompasses genes that have evolved at a distinct evolutionary stage (for example, a set of genes might be common to all cells, to all animals or to all chordates). By overlaying this gene classification on the developmental transcriptome of zebrafish (Danio rerio), they showed that genes that are expressed at the phylotypic stage belong to the oldest and most conserved set; by contrast, genes expressed earlier or later are more likely to belong to more newly evolved gene sets. A similar pattern was also seen in published data from Drosophila melanogaster, Anopheles gambiae and Caenorhabditis elegans.

The phylotypic stage is the step at which the principal animal body-plan is laid down - the conserved expression profile described by these studies would have been established early in the evolution of multicellular life, and the steps both before and after it would have diverged to forge the morphological diversity we see across animal species. However, the mechanism that preserves the expression profile seen in the hourglass waist remains to be definitively established: is the mid-embryonic stage actively constrained by selection or is it under less pressure to evolve than early and late stages? These studies open up opportunities to address such mechanistic questions experimentally.

Tanita Casci

ORIGINAL RESEARCH PAPERS Kalinka, A. T. et al. Gene expression divergence recapitulates the developmental hourglass model. Nature $\mathbf{4 6 8}$, 811-814 (2010)| Domazet-Lošo, T. \& Tautz, D. A phylogenetically based transcriptome age index mirrors ontogenetic divergence patterns. Nature 468, 815-818 (2010) 